\title{
Velocity autocorrelations of decaying isotropic homogeneous turbulence
}

\author{
Mei-Jiau Huang \\ Institute of Physics, Academia Sinica, Taiwan \\ Anthony Leonard \\ Graduate Aeronautical Laboratories, California Institute of Technology, Pasadena, California 91125
}

(Received 28 December 1994; accepted 12 June 1995)

\begin{abstract}
Velocity autocorrelations and the mean-square displacements of fluid particles are obtained for decaying, isotropic homogeneous turbulence by numerical simulation of the flow field, using $128^{3}$ and $256^{3}$ grids, and tracking several tens of thousands of fluid particles, using a third-order interpolation scheme. A self-preserving Lagrangian velocity autocorrelation coefficient is found in terms of a dimensionless time variable $s$, defined by $d s=d t / \mathscr{F}_{s}(t)$, under the observation of a power-law energy decay and the assumption that $\mathscr{T}_{s}(t)$ is proportional to the Lagrangian integral timescale $\mathscr{F}_{\mathscr{P}}$. This timescale is in turn assumed to be proportional to the length scale of the energy-containing eddies $\mathscr{L}_{e} \sim K^{3 / 2} / \epsilon$ divided by the turbulent velocity $u^{\prime}$, where $K=\frac{3}{2} u^{\prime 2}$ is turbulent energy and $\epsilon$ is the energy dissipation rate. (C) 1995 American Institute of Physics.
\end{abstract}

\section{INTRODUCTION}

In the study of turbulent dispersion, the use of Lagrangian coordinates is more natural than the use of fixed or Eulerian coordinates. Unfortunately, Lagrangian statistics are much less accessible and even in numerical simulations additional complications arise in their determination. However, some techniques have been developed to obtain useful data. By measuring the dispersion of thermal fluctuations behind a heated wire in a grid-generated turbulent flow and based on 'Taylor's classic work on diffusion of $1921,{ }^{1}$ Shlien and Corrsin $^{2}$ were able to determine Lagrangian velocity autocorrelations. Sato and Yamamoto ${ }^{3}$ used an optical tracer-particle tracking method to measure Lagrangian velocity autocorrelations directly in a grid-generated turbulent flow. The spread of the mean and fluctuating thermal field for line sources has also been investigated, for example, by Uberoi and Corr $\sin ,{ }^{4}$ Townsend, ${ }^{5}$ Stapountzis et al. ${ }^{6}$ and Warhaft. ${ }^{7}$ All these measurements take into account the decay of turbulence to some extent.

An alternative to extract Lagrangian information is the use of direct numerical simulations (DNS). Riley and Patterson $^{8}$ were the first to calculate Lagrangian statistics in the DNS of decaying turbulence. The fluid velocity at the particle position (a total of 432 particles were tracked) was computed from a linear interpolation of the values of the Eulerian velocity field. Later Yeung and Pope ${ }^{9}$ argued that a higher order interpolation scheme should be used for tracking in a turbulent flowfield and so employed a third-order accurate Taylor series scheme to measure the Lagrangian structure functions in stationary turbulence. A cubic spline interpolation scheme was used subsequently by Yeung and Pope $^{10}$ to obtain high-order quantities such as Lagrangian acceleration and velocity gradients. On the other hand Gotoh et al. " used the so-called passive vector method in which explicit velocity interpolation was avoided by transporting the initial velocity as a passive vector. The method is found to be accurate when the ratio of the maximum resolvable wavenumber to Kolmogorov wavenumber is greater than 2 but is limited to relatively short times because the passive vector has zero diffusivity. In other numerical simulations Lee et $_{\text {al. }}{ }^{12}$ and Squires and Eaton ${ }^{13}$ have investigated dispersion of fluid particles in decaying isotropic turbulence and in homogeneous shear flows using particle tracking.

Theoretical studies of Lagrangian statistics includes Corrsin's ${ }^{14}$ application of Kolmogorov similarity to both Lagrangian and Eulerian frequency spectra. Lagrangian and Eulerian scales were shown to be of the same order. On the other hand, Tennekes ${ }^{15}$ proposed an "advection concepl" in which the dissipation eddies are advected by large eddies relative to an Eulerian observor. As a consequence it can be argued that the Eulerian frequency spectrum extends to higher frequencies than its Lagrangian counterpart. Using Corrsin's "independence" conjecture ${ }^{16}$ (viz., for large time, the distribution of particle displacements becomes statistically independent of the particular realization of the velocity field), Saffman ${ }^{17}$ derived an intego-differential equation for Lagrangian velocity autocorrelation in stationary turbulence. Assuming a Gaussian distribution of particle displacements, he found that the Lagrangian velocity autocorrelation may decay algebraically at large times.

Another theory for particle dispersion in decaying turbulence is based on the hypothesis of a self-preserving Lagrangian velocity autocorrelation (Batchelor and Townsend ${ }^{18}$ ). Given a power-law decay of turbulence energy, one assumes that an appropriately modified velocity is a stationary random function of a new time variable defined by a characteristic timescale. A consequence of the hypothesis is that the Lagrangian velocity autocorrelation coefficient becomes selfpreserving in the new time variable. The theory has been testcd by Sato and Yamamoto ${ }^{3}$ who chose a characteristic timescale, $\Lambda_{f} / u^{\prime}$, where $\Lambda_{f}$ is the longitudinal integral lengthscale. Using several empirical relations, they found a self-preserving Lagrangian coefficient with an exponential tail in the new time variable. Shlien and Corrsin, ${ }^{2}$ using a measured exponent of 1.25 for the decay of turbulent energy and making several assumptions, obtained a new time vari- 
able that was the same as that of Sato and Yamamoto. Using Taylor's hypothesis, they were then able to calculate the Lagrangian velocity correlation coefficient from the measured dispersion data.

In the present study, we use the methods of Yeung and Pope to study numerically particle dispersion problems in decaying, approximately isolropic, homogeneous turbulence, computed by a Fourier spectral method. We reexamine the validity above-mentioned hypotheses and attempt to find a solid basis for an appropriately scaled time variable, leading to self-preservation of the Lagrangian correlation coefficient. This paper is organized as follows. The numerical methods for determining the evolution of the velocity field and for particle tracking are described in Sec. II, along with a discussion of the observed power-law energy decay. In Section III various forms of velocity autocorrelation coelficients are defined and their means of computation are given. Numerical results are given in Sec. IV, including measurements of microscales (time and length), comparisons of Lagrangian and Eulerian velocity autocorrelation coefficients, the selfpreservation of Lagrangian velocity autocorrelation coefficient, and its application in predicting the mean-square displacement of fluid particles. Conclusions and a discussion of the results are given in Sec. V.

\section{NUMERICAL METHODS}

\section{A. Eulerian fields}

We investigate the Eulerian and Lagrangian statistics of decaying turbulence governed by the incompressible NavierStokes equations,

$$
\begin{aligned}
& \frac{\partial \mathbf{u}}{\partial t}+\mathbf{u} \cdot \nabla \mathbf{u}=-\nabla p+\nu \nabla^{2} \mathbf{u} \\
& \nabla \cdot \mathbf{u}=0,
\end{aligned}
$$

where $\mathbf{u}$ is the turbulent velocity, $p$ is the pressure, $\nu$ is the kinematic viscosity, and the density $\rho \equiv 1$. The flow is as sumed to be confined within a periodic physical domain $I_{o}^{3}$ and thus can be represented by a Fourier series expansion. In the present study, we use the algorithm developed by Rogallo $^{19}$ for homogeneous turbulence. The Navier-Stokes equations are solved by the Fourier pseudo-spectral method and integrated in time by a second-order Runge-Kutta method. The induced aliasing errors are nearly eliminated by a combination of truncation and use of a phase-shifting technique. ${ }^{19,20}$ The phase shifting technique consists of evaluating the velocity products. in the space $\mathbf{x}+\frac{1}{2} \Delta \mathbf{x}$ at the predictor step and in the space $\mathbf{x}$ at the corrector step, so that the aliasing error from the predictor step almost cancels the error from the corrector step. For a further improvement, the phase shifts are chosen at random on each time substep. In our simulations, there is no external forcing and the small eddies contain little energy. The remaining aliasing errors are thus expected to be negligible.

If $\hat{u}_{i}(\mathbf{k}, t)$ is the $i$-component of velocity Fourier coefficient of wave vector $\mathbf{k}$ at time $t$, we find that $\hat{u}_{i}$ evolves according to

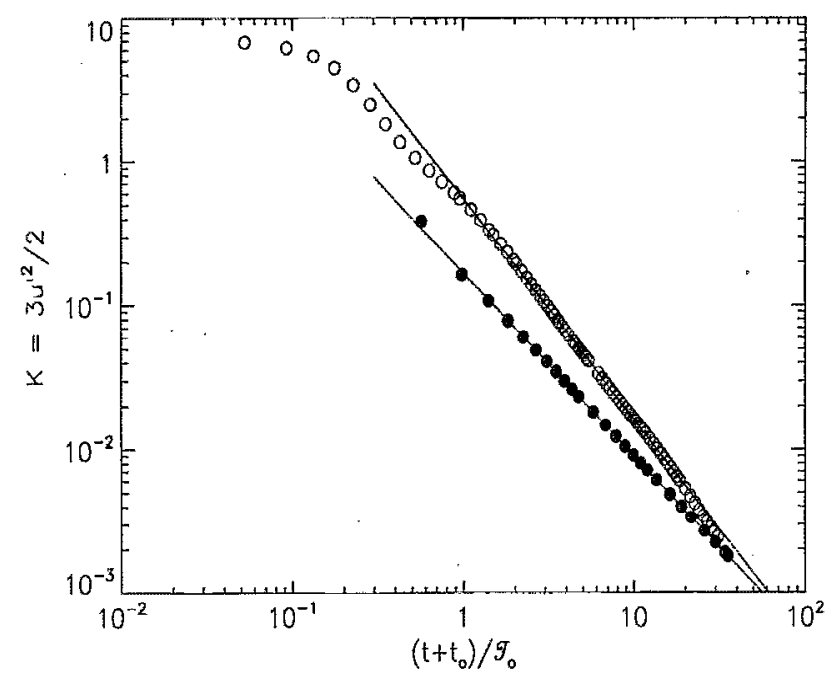

FIG. 1. Decay of turbulent energy $\mathrm{K}$. Time is nondimensionalized by the eddy turnover time $\mathscr{F}_{0}$ at $k_{\max } \eta \approx 1.0$, run $1 ; \bullet$, run 2 .

$$
\frac{\partial \widehat{u}_{i}}{\partial t}+\left(\delta_{i j}-k_{i} k_{j} / k^{2}\right) \cdot i k_{m}\left(\widehat{u_{m} u_{j}}\right)+\nu k^{2} \widehat{u_{i}}=0,
$$

where $\delta_{i j}$ is the Kronecker delta and $\widehat{u_{m} u_{j}}$ is the Fourier coefficient of velocity product $u_{m} u_{j}$. The Fourier coefficients of the velocity products $\left(\widehat{u_{m} u_{j}}\right)$ are evaluated by using the pseudo-spectral method, modified as described above.

Two simulations of decaying homogeneous and nominally isotropic turbulence are performed. The initial velocity fields are constructed in wave space in a way so that the continuity condition is satisfied and the initial energy spectrum is specified. ${ }^{19}$ The initial energy spectra are chosen from

$E(k, 0)=\left\{\begin{array}{l}C u_{o}^{2} \exp (-a)\left(k / k_{p}\right)^{2}, \quad \text { for } 0 \leqslant k \leqslant k_{p} ; \\ C u_{o}^{2}\left(k / k_{p}\right)^{-5 / 3} \exp \left(-a k / k_{p}\right), \quad \text { for } k_{p} \leqslant k ;\end{array}\right.$

and

$$
E(k, 0)=\frac{3}{2} \frac{u_{o}^{2}}{k_{p}}\left(\frac{2 k}{k_{p}}\right)^{2} \exp \left(-\frac{2 k}{k_{p}}\right),
$$

where $C$ is chosen so that the initial mean kinetic energy is $\frac{3}{2} u_{o}^{2}$ and the initial energy spectra have a peak at $k=k_{p}$. The values $a=5.1$ and $u_{0}=1$ are used.

Before fluid particles are released, the velocity field is allowed to decay from the initial conditions to a developed state in which the turbulent kinetic energy decays according to a power law (Huang and Leonard ${ }^{21}$ ). The evolution in time of the energy and of the Reynolds number based on the Taylor's microscale $\lambda, \operatorname{Re}_{\lambda}=u^{\prime} \lambda / \nu$, are shown in Figs. 1 and 2, respectively. The Reynolds numbers of these two cases are about in the same ranges and are about 42 when the powerlaw decay begins. At this point we also have $k_{\max } \eta \approx 1$, where $k_{\max }=(\sqrt{2} N / 3) \cdot(2 \pi / L)(N$ is the resolution, i.e., the number of grid points in each direction) is the maximum resolvable wavenumber and $\eta$ is the Kolmogorov dissipation lengthscale. The decay exponents are found to be about 1.5 


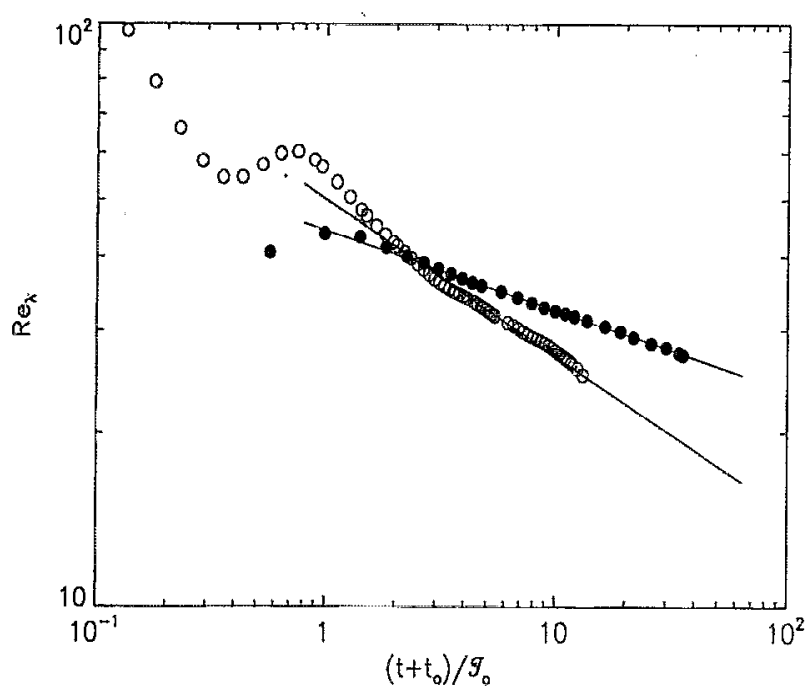

FIG. 2. Decay of Reynolds number $\operatorname{Re}_{\lambda}$. Time is nondimensionalized by the eddy turnover lime $\mathscr{P}_{n}$ at $k_{\max } \eta \approx 1$. 0 , run $1 ; 0$, run 2 .

in one case and 1.3 in the other. These two cases are representative of a larger set of cases studied previously by Huang and Leonard. ${ }^{21}$ Initial flow information and some important dynamic information of the flows when $k_{\max } \eta \approx 1$ can be found in Table I. Note the eddy-turnover time $\mathscr{T}_{o}$ is defined as

$$
\mathscr{F}_{0} \equiv \frac{3 \pi}{4 u^{\prime}} \int_{0}^{\infty} k^{-1} E(k, t) d k / \int_{0}^{\infty} E(k, t) d k
$$

and the Kolmogorov dissipation time scale $\tau_{\eta}$ is defined as

$$
\tau_{\eta} \equiv \eta^{2} / \nu=(\nu / \epsilon)^{1 / 2} \text {. }
$$

\section{B. Lagrangian variables}

A fluid particle is defined as a mathematical point moving with the local velocity of the fluid continuum. Let $\boldsymbol{\xi}$ be the initial position of a fluid particle and $\mathbf{x}_{p}$ be the position of the particle at time $t$. A fluid particle is thus tracked by solving

$$
\frac{d \mathbf{x}_{p}(t)}{d t}=\mathbf{u}\left(\mathbf{x}_{p}, t\right), \text { with } \mathbf{x}_{p}(0)=\xi
$$

TABLE I. Eulerian velocity fields.

\begin{tabular}{lcccccc}
\hline \hline & \multicolumn{2}{c}{ Initial conditions } & \multicolumn{2}{c}{$k_{\max } \eta \approx 1$} & \multicolumn{2}{c}{$k_{\max } \eta \approx 2(t \equiv 0)$} \\
& Run 1 & Run 2 & Run 1 & Run 2 & Run 1 & Run 2 \\
\hline$E(k, 0)$ & Eq. (4) & Eq. (5) & $\ldots$ & $\ldots$ & $\ldots$ & $\ldots$ \\
$k_{p} L_{n}$ & $8 \pi$ & $24 \pi$ & $\ldots$ & $\ldots$ & $\ldots$ & $\ldots$ \\
$\nu$ & 0.002 & 0.0013 & $\ldots$ & $\ldots$ & $\ldots$ & $\ldots$ \\
$\mathrm{N}$ & 128 & 256 & $\ldots$ & $\ldots$ & $\ldots$ & $\ldots$ \\
$\mathrm{M}$ & 16,384 & 30,000 & $\ldots$ & $\ldots$ & $\ldots$ & $\ldots$ \\
$R e_{\lambda}$ & 203.1 & 165.5 & 42.3 & 42.6 & 32.8 & 35.7 \\
$\lambda / L_{n}$ & $4.3 \%$ & $1.7 \%$ & $3.6 \%$ & $1.7 \%$ & $5.7 \%$ & $3.0 \%$ \\
$\tau_{\eta}$ & 0.022 & 0.055 & 0.16 & 0.22 & 0.51 & 0.77 \\
$\mathscr{F}_{*} / \tau_{\eta}$ & 10.0 & 7.3 & 13.1 & 13.1 & 11.4 & 10.7 \\
\hline \hline
\end{tabular}

In the present study, we solve ( 8 ) by using the same timedifferencing method used to evolve the velocity field, a second-order Rúnge-Kutta method. As discussed above, a phase-shift technique is used to minimize the aliasing error in evolving the Navier-Stokes equations, ${ }^{19}$ and this must be kept in mind when integrating the particle equations. Specifically, at the predictor step, the grid for the velocity field has been shifted by $\mathbf{x}+\frac{1}{2} \Delta \mathbf{x}+\mathbf{r}_{1}$ and at the corrector step by $\mathbf{x}+\mathbf{r}_{2}$, where $\mathbf{r}_{1}$ and $\mathbf{r}_{2}$ are random vectors uniformly distributed within $(0, \Delta \mathbf{x})$. Interpolation methods are then used to estimate the velocities at the particles' positions $\mathbf{x}_{p}$ at both steps. The results are then used to time march Eq. (8).

The accuracies of a variety of interpolation methods have been investigated by Yeung and Pope. ${ }^{9}$ They found that the second-order accurate, linear interpolation method, using eight grid points, is unsatisfactory for turbulent flows, because, in general, there is significant energy at the higher wavenumbers. Here we use 13-point third-order scheme that is a modification of the so-called T13 method suggested by Yeung and Pope for planewise data sets. The induced interpolation errors were found to be within tolerance. ${ }^{9}$ However, in the original T13 method velocities at the grid points $\mathbf{x}_{i}$ and $\mathbf{x}_{i}+\frac{1}{2} \Delta \mathbf{x}$ would be needed, where $\mathbf{x}_{i}$ are $\mathbf{x}+\frac{1}{2} \Delta \mathbf{x}+\mathbf{r}_{1}$ or $\mathbf{x}+\mathbf{r}_{2}$ when random grid shifts are employed. Thus additional FFTs and memory space would be required. To avoid these additional requirements we modify the scheme so that only velocities at grid points $\mathbf{x}_{i}$ are needed and the scheme remains third-order accurate. The modified formula is given in the Appendix.

It has been found (Yeung and Pope'; Eswaran and Pope $^{22}$ ) that the spatial resolution is to a significant extent determined by the value of $k_{\max } \eta$. To test the accuracy of the modified T13 scheme, we apply the modified scheme to one of our decaying turbulent flows at several times during the decay. The velocities at $\mathbf{x}_{i}+\frac{1}{2} \Delta \mathbf{x}$ in a certain plane are evaluated by both the modified T13 scheme and the FFT. The absolute differences are then averaged over all the points evaluated and then normalized by the rms velocity $u^{\prime}$. The results are called "normalized errors" and presented in Fig. 3 . Note that we observe third-order accuracy as $k_{\max } \eta$ increases. The results suggest that a minimum value of $k_{\max } \eta \approx 1.5$ is required to obtain an error under $3 \%$. In the present simulations, we thus introduce particles into the flow as $k_{\max } \eta \approx 2$, at which the time variable $(t)$ is assigned zero. The accuracy at later times is expected to be even better because, unlike stationary turbulence, the eddies grow in size and $k_{\max } \eta$ increases as time increases. Information on the state of the flow at $k_{\max } \eta \approx 2(t=0)$ can be found in Table I. The CPU seconds per time step required using $\mathrm{N}$ nodes (i.e., the number of nodes used equals the resolution in each direction) of the Intel Delta parallel computer with $M$ particles are listed in Table II.

It is known that the sampling error is proportional to $M^{-1 / 2}$. We were able to track $128^{2}=16,384$ and 30,000 particles in run 1 and run 2 , respectively, whereas earlier studies employed roughly $400^{8}$ to 4000 particles. ${ }^{10,13}$ Compared with interpolation errors and temporal errors [which is determined by the Courant number $C_{o} \equiv \Delta t N\left(\left|u_{1}\right|_{\max }+\left|u_{2}\right|_{\max }\right.$ $\left.+\left|u_{3}\right|_{\text {max }}\right) / L_{o}$; while $C_{o} \leqslant 1 / 2 \pi$ is used in the present simu- 


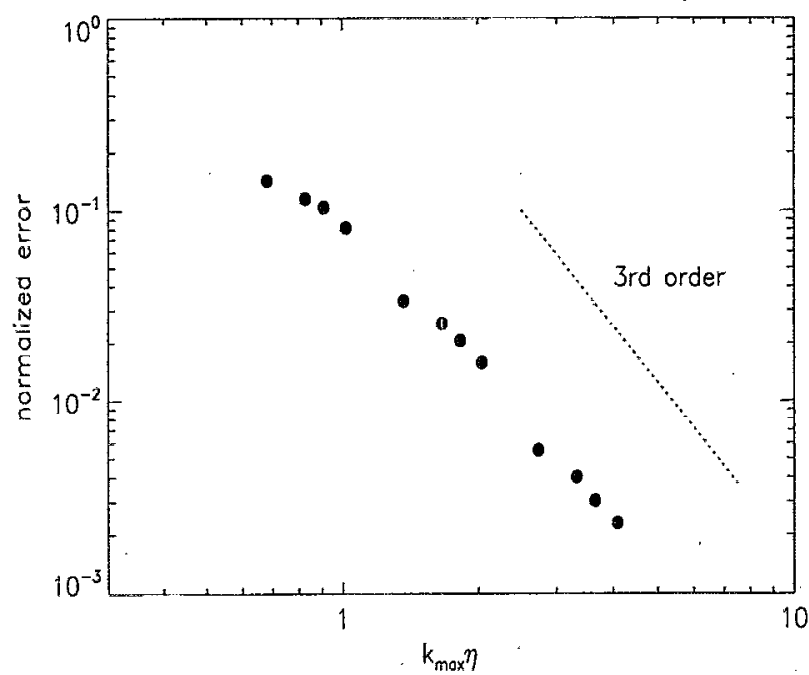

FIG. 3. Interpolation errors of the modified T13 scheme on turbulent velocity fields.

lations]. the sampling errors are believed to be relatively small. $^{23}$

\section{EXTRACTION OF EULERIAN AND LAGRANGIAN DATA}

We study the double velocity autocorrelation coefficients, including the two-time-one-point, $f_{E}\left(t, t^{\prime}\right)$, and onetime-two-point Eulerian coefficients, $f(r, t)$, as well as the Lagrangian coefficient, $f_{L}\left(t, t^{\prime}\right)$. By definition, they are in homogeneous isotropic turbulence,

$$
\begin{aligned}
& f(r, t)=\frac{1}{3} \sum_{\alpha=1}^{3} \overline{u_{\alpha}(\mathbf{x}, t) u_{\alpha}\left(\mathbf{x}+r \mathbf{e}_{\alpha}, t\right)} / \overline{u_{\alpha}^{2}}(t), \\
& f_{E}\left(t^{\prime}, t\right)=\frac{1}{3} \sum_{\alpha=1}^{3} \overline{u_{\alpha}(\mathbf{x}, t) u_{\alpha}\left(\mathbf{x}, t^{\prime}\right)} / \sqrt{\overline{u_{\alpha}^{2}}(t) \overline{u_{\alpha}^{2}}\left(t^{\prime}\right),}
\end{aligned}
$$

and

$$
f_{L}\left(t^{\prime}, t\right)=\frac{1}{3} \sum_{\alpha=1}^{3}\left\langle u_{\alpha}(\xi, t) u_{\alpha}\left(\xi, t^{\prime}\right)\right\rangle / \sqrt{\overline{u_{\alpha}^{2}}(t) \overline{u_{\alpha}^{2}}\left(t^{\prime}\right),}
$$

where $\mathbf{e}_{\alpha}$ is the unit vector in the $\alpha$ direction, the overbar represents a volume average, and $\langle\cdot\rangle$ is an ensemble average over the particles released, i.e. a Lagrangian average. Recall that $t$ and $t^{\prime}$ are measured from the time at which particles were released. With the velocity Fourier coefficients at hand, we compute $f_{E}$ as follows:

TABLE II. CPU seconds required per time step.

\begin{tabular}{ccccc}
\hline \hline NM & 0 & 4096 & 16,384 & 30,000 \\
\hline 128 & 1.61 & 2.63 & 4.50 & $\ldots$ \\
256 & 7.96 & $\cdots$ & 11.15 & 13.17 \\
\hline
\end{tabular}

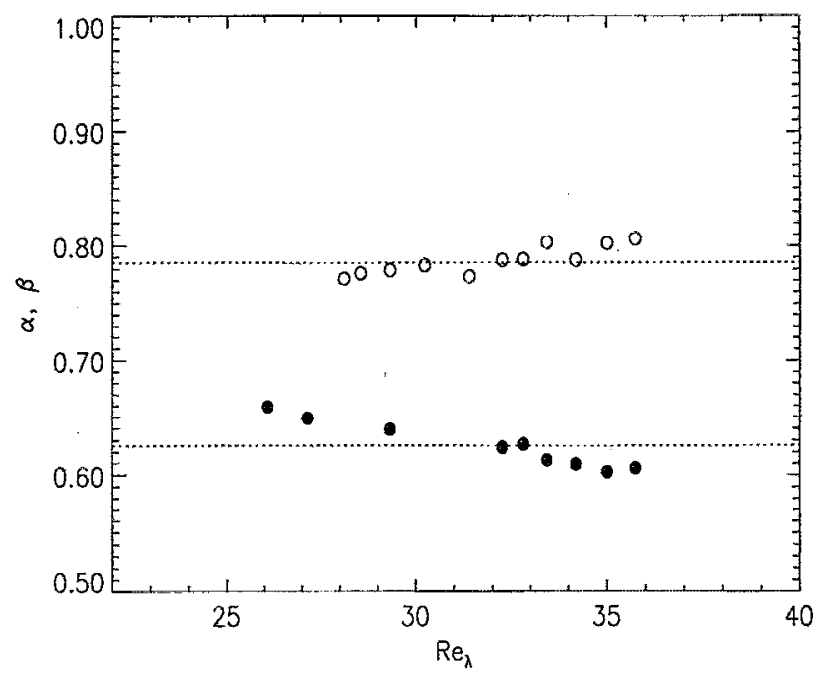

FIG. 4. Nondimensional dissipation timescales. $\quad \alpha=u^{\prime} \tau_{E} / \lambda ; 0$, $\beta=u^{r} \tau_{L} / \lambda$. Horizontal lines are the averages.

$f_{E}\left(t^{\prime}, t\right)=\frac{1}{3} \sum_{\alpha=1}^{3}\left(\sum_{\mathbf{k}} \hat{u}_{\alpha}(\mathbf{k}, t) \hat{u}_{\alpha}^{*}\left(\mathbf{k}, t^{\prime}\right)\right) / \sqrt{\sqrt{\tilde{u}_{\alpha}^{2}(t) \vec{u}_{\alpha}^{2}\left(t^{\prime}\right)},}$

which is symmetric in $t$ and $t^{t}$ because the velocity is real, and

$$
\begin{aligned}
f(r, t)= & \frac{1}{3} \sum_{\alpha=1}^{3}\left\{\sum_{\hat{k}_{\alpha}}\left(\sum_{\substack{k_{j} \\
j \neq \alpha}} \hat{u}_{\alpha}(\mathbf{k}, t) \overline{u_{\alpha}^{*}}(\mathbf{k}, t)\right)\right. \\
& \left.\times \exp \left(-i k_{\alpha} r\right)\right\} / \overline{u_{\alpha}^{2}}(t),
\end{aligned}
$$

where "*" means the complex conjugate.

\section{RESULTS}

\section{A. Comparison of dissipation scales}

We consider three dissipation time/length scalesTaylor's microscale $(\lambda)$, the Eulerian and Lagrangian dissipation timescales $\left(\tau_{E}\right.$ and $\left.\tau_{L}\right)$, defined by the following:

$$
\begin{aligned}
& \lambda^{-2}=-\left.\frac{\partial^{2} f(r, t)}{\partial r^{2}}\right|_{r=0}, \quad \tau_{E}^{-2}=-\left.\frac{\partial^{2} f_{E}}{\partial t^{\prime 2}}\right|_{t^{\prime}=t}, \\
& \tau_{L}^{-2}=-\left.\frac{\partial^{2} f_{L}}{\partial t^{\prime 2}}\right|_{t^{\prime}=t} .
\end{aligned}
$$

In the present study, the temporal scales are computed by measuring the curvatures of the correlation coefficients using a fourth-order finite difference method and assuming a parabolic behavior near the origin; while the l'aylor scale is found from $\lambda^{2}=15 \nu u^{\prime 2} / \epsilon$. In Fig. 4, we show the nondimensional timescales $\alpha(t) \equiv u^{\prime} \tau_{E} / \lambda$ and $\beta(t)=u^{\prime} \tau_{L} / \lambda$ as a function of $R e_{\lambda}$. A slight increase of $\alpha$ and a slight decrease of $\beta$ with decreasing $R_{\lambda}$ are observed. Note that $\alpha<\beta$ implies that $f_{E}<f_{L}$ at small time lag $\left(t^{\prime}-t\right)$. The same result 

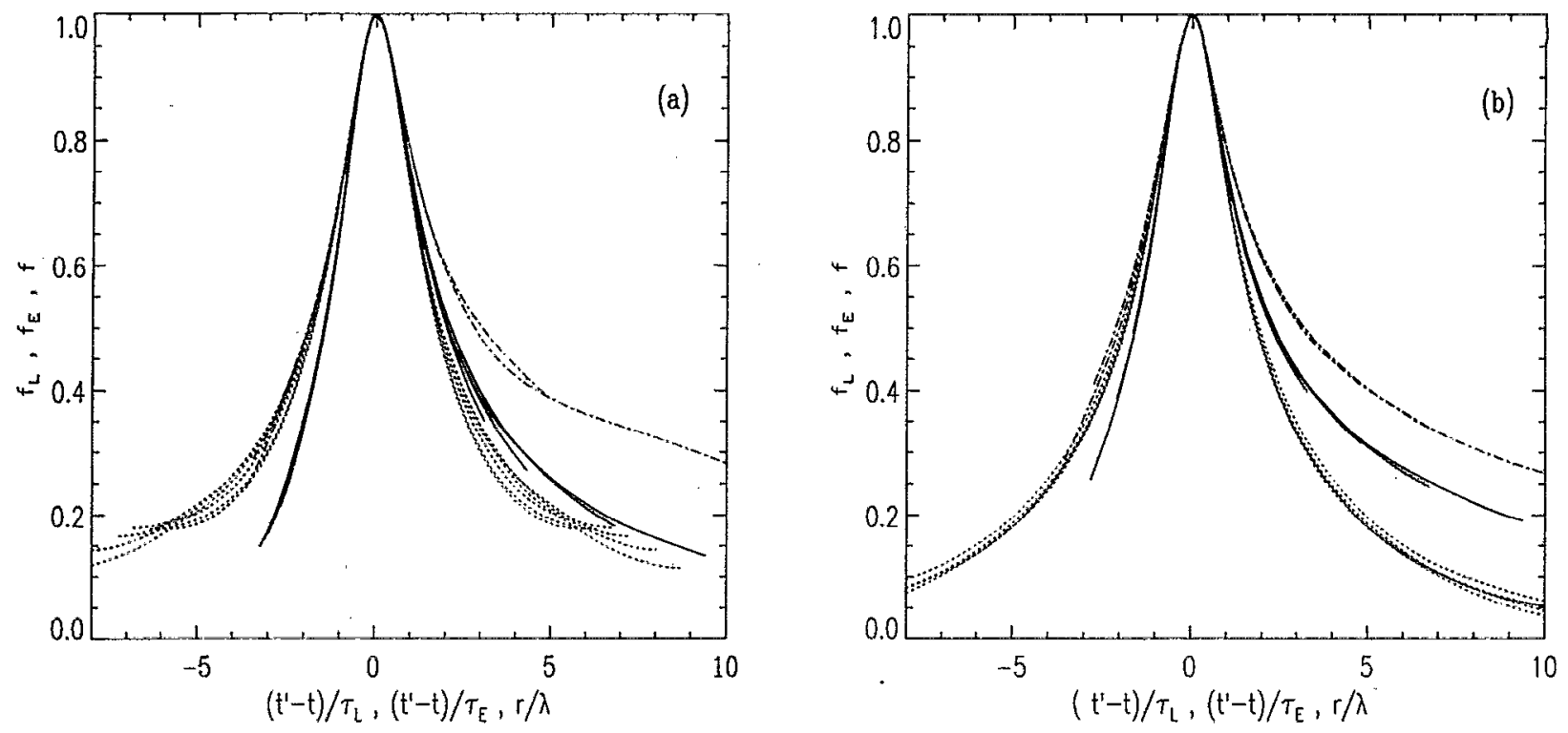

FIG. 5. Velocity autocorrelation coefficients at several times after release for (a) run 1 ; (b) run 2. -,$f_{L}\left(t, t^{\prime}\right)$ vs $\left(t^{\prime}-t\right) / \tau_{L} ;-\cdot-, f_{E}\left(t, t^{\prime}\right)$ vs $\left(t^{\prime}-t\right) / \tau_{E} ; \cdots f(r, t)$ vs $r / \lambda$.

was also observed by Rily and Patterson ${ }^{8}$ and Shlien and Corrsin. ${ }^{2}$ The averaged value of $\alpha$ is about 0.624 within the range of $R e_{\lambda}$ investigated, which is very close to the value of $\sqrt{2 / 5} \approx 0.632$ predicted by Tennekes in $1975^{15}$ based on the Taylor's frozen hypothesis and the assumption that the energy-containing eddies and the dissipation eddies are statistically independent in isotropic homogeneous turbulence.

In Fig. 5, we show together the correlation coefficients $f(r, t), f_{E}\left(t, t^{\prime}\right)$, and $f_{L}\left(t, t^{\prime}\right)$. vs $r / \lambda,\left(t^{\prime}-t\right) / \tau_{E}$, and $\left(t^{\prime}-t\right) / \tau_{L}$, respectively, at several times. The temporal correlation coefficients, $f_{E}$ and $f_{L}$, are non-symmetric because the flow is decaying; while the spatial coefficient $f(r, t)$ is symmetric since the flow is homogeneous. The collapse of the curves at small time lags is simply a result of parabolic behavior of the $f$ 's at small values of their respective arguments.

As shown, both $f_{L}$ and $f_{E}$ have larger tails at positive $\left(t^{\prime}-t\right)$ than at negative $\left(t^{\prime}-t\right)$ and the tails of $f_{E}$ are larger than those of $f_{L}$. The observation that $f(r, t)$ vs $r / \lambda$ is nearly equal to $f_{E}\left(t, t^{\prime}\right)$ vs $\left(t^{\prime}-t\right) / \tau_{E}$ at the same time $t$ for negative time lags suggests the possibility of a transformation between the two-time-one-point and one-time-two-point Eulerian velocity autocorrelation coefficients.

\section{B. Self-preservation of $f_{L}\left(t^{\prime}, t\right)$}

In stationary turbulence, $f_{E}$ and $f_{L}$ are functions only of $\left|t^{\prime}-t\right|$. In decaying turbulence, Batchelor and Townsend ${ }^{18}$ were first to argue that a self-preserving Lagrangian velocity autocorrelation coefficient is also possible. We now give a brief description of Batchelor and Townsend's selfpreserving hypothesis and examine it with respect to the present numerical results. The hypothesis states that, if the turbulent energy decays according to a power law,

$$
K \sim\left(t+t_{o}\right)^{-n}
$$

where $t_{0}$ is a virtual time origin, then there exists some characteristic time scale, $\mathscr{F}_{s}(t)$, such that $u(t)\left(1+t / t_{o}\right)^{n / 2}$, where $u(t)$ is the particle velocity, is a stationary random function of a new time variable $s$, defined by $d s \equiv d t / \mathscr{T}_{s}$. Following the hypothesis, the Lagrangian correlation coefficient must be self-preserving in this new time variable $s$, since

$$
\begin{aligned}
f_{L}\left(t^{\prime}, t\right) & \equiv \frac{\left\langle u(t) u\left(t^{\prime}\right)\right\rangle}{u^{\prime}(t) u^{\prime}\left(t^{\prime}\right)} \\
& =\frac{\left\langle\left(u(t)\left(1+t / t_{o}\right)^{n / 2}\right)\left(u\left(t^{\prime}\right)\left(1+t^{\prime} / t_{o}\right)^{n / 2}\right)\right\rangle}{\left(u^{\prime}(t)\left(1+t / t_{o}\right)^{n / 2}\right)\left(u^{\prime}\left(t^{\prime}\right)\left(1+t^{\prime} / t_{o}\right)^{n / 2}\right)} \\
& -\frac{\left\langle\tilde{u}(s) \tilde{u}\left(s^{\prime}\right)\right\rangle}{\widetilde{u}^{\prime}(s) \widetilde{u}^{\prime}\left(s^{\prime}\right)}-\tilde{\int}_{L}\left(s^{\prime}-s\right)-\tilde{S}_{L}\left(s^{\prime \prime}\right),
\end{aligned}
$$

where $\quad s^{\prime \prime}=s^{\prime}-s, \quad \tilde{u}(s(t)) \equiv u(t)\left(1+t / t_{o}\right)^{n / 2}, \quad$ and $\widetilde{u^{\prime}}(t) \equiv u^{\prime}(s(t))\left(1+t / t_{o}\right)^{n / 2}$. Moreover, $\tilde{f}_{L}\left(s^{\prime \prime}\right)$ is symmetric, i.e. $\tilde{f}_{L}\left(-s^{\prime \prime}\right)=\tilde{f}_{L}\left(s^{\prime \prime}\right)$, because $f_{L}\left(t, t^{\prime}\right)=f_{L}\left(t^{\prime}, t\right)$.

The power-law energy decay has been predicted by a variety of theories and supported by numerical as well as experimental data (see Refs. 21, 24-26, etc.) We consider now the determination of $\mathscr{F}_{s}$. It should, as a consequence of the hypothesis, be related to the Lagrangian integral time scale $\mathscr{T}_{L}$ by

$$
\begin{aligned}
\mathscr{F}_{L}(t) & \equiv \int_{t}^{\infty} f_{L}\left(t, t^{\prime}\right) d t^{\prime} \\
& =\int_{s}^{\infty} f_{L}\left(t, t^{\prime}\right) \mathscr{F}_{s}\left(t^{\prime}\right) d s^{\prime} \\
& =\int_{0}^{\infty} \mathscr{F}_{s}\left(t^{\prime}\right) \tilde{f}_{L}\left(s^{\prime \prime}\right) d s^{\prime \prime} .
\end{aligned}
$$



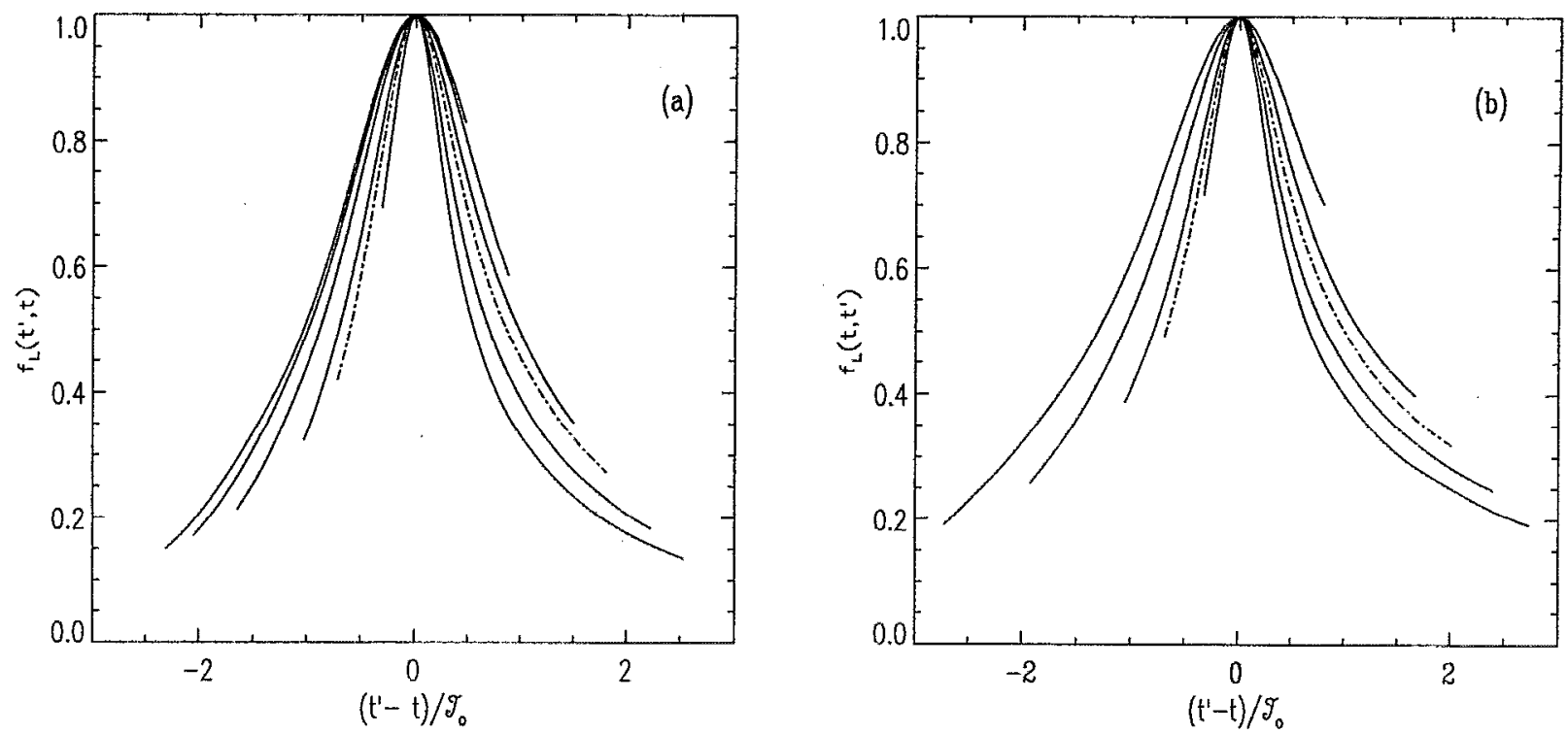

FIG. 6. Lagrangian velocity autocorrelation coefficients $f_{L}\left(t, t^{\prime}\right)$ vs $\left(t^{\prime}-t\right) \mathscr{F}_{o}$, where $\mathscr{F}_{s}$ is eddy turnover time at $k_{\max } \eta 2$ ( $(t=0)$, for (a) run 1 , (b) run 2 at several times after release. The dash-dotted curve corresponds to one particular time.

Assuming $n=1$ and $\mathscr{F}_{s}(t) \sim \lambda / u^{\prime}$, Batchelor and Townsend found that $\mathscr{T}_{s}=t+t_{0}$. Sato and Yamamoto ${ }^{27}$ obtained the same result but based on the assumption that $\mathscr{T}_{s} \sim \mathscr{F}_{L}$ and the empirical relations of $u^{\prime} \mathscr{F}_{L} \sim \mathscr{L}_{f}$ and $\mathscr{Z}_{f} / \lambda \sim R e_{\lambda}$, where $\mathscr{L}_{f}$ is the longitudinal integral lengthscale, i.e., the integral of $f(r, t)$ from $r=0$ to infinity. Consequently, $s=\log \left(1+t / t_{o}\right)$.

However, we need to reconsider the above arguments leading to the determination of $\mathscr{F}_{s}$. For example the decay exponent $n=1$ is not observed in experiment or simulation (e.g., $n=1.3$ and 1.5 are observed in the present simulations) and for general $n$, Batchelor and Townsend's assumption would lead to $\mathscr{F}_{s}=\left(t+t_{o}\right)^{(n+1) / 2}$. In addition, the empirical relations used by Sato and Yamamoto are not well supported by experimental data. (The ratio $u^{\prime} \mathscr{F}_{L} / \mathscr{L}_{f}$ is, in general, observed to be $R e_{\lambda}$-dependent as seen in their own experimental data. $^{3}$ In addition, we believe that the ratio $\mathscr{L}_{f} / \lambda \approx A+B R e_{\lambda}^{\beta}$ for $R e_{\lambda}$ small, where $A, B$ and $\beta$ are all constants, ${ }^{21}$ while $\mathscr{B}_{f} / \lambda \approx$ constant as $\operatorname{Re}_{\lambda} \gg 1$ considering the von Kármán-Howarth similarity. ${ }^{26}$ ) However, we show that the choice $\mathscr{T}_{s} \sim \mathscr{F}_{L}$ is a good one in that it leads to a consistent hypothesis. In addition, at very high Reynolds number, we may approximate energy spectrum by

$$
E(k)=\left\{\begin{array}{l}
0, \quad \text { for } k<k_{o} \equiv \mathscr{L}_{e}^{-1} ; \\
C \epsilon^{2 / 3} k^{-5 / 3}, \quad \text { for } k_{o} \leqslant k \leqslant k_{\eta} \equiv \eta^{-1} ; \\
0, \text { for } k_{\eta}<k ;
\end{array}\right.
$$

and the Lagrangian frequency spectrum $\phi_{L}(\omega)$ [the Fourier Iransform of Lagrangian velocity correlation $\overline{u(\xi, t) u\left(\xi, t^{\prime}\right.}$ with respect to variable $t^{\prime}-t$, where $\omega$ is frequency] by

$\phi_{L}(\omega)=\left\{\begin{array}{l}0, \text { for } \omega<\omega_{o} \equiv \mathscr{F}_{L}^{-1} ; \\ B \epsilon \omega^{-2}, \quad \text { for } \omega_{o} \leqslant \omega \leqslant \omega_{\eta} \equiv(\epsilon / \nu)^{1 / 2} ; \\ 0, \text { for } \omega_{\eta}<\omega ;\end{array}\right.$ where $\mathscr{B}_{e}$ is the lengthscale characterizing the energycontaining eddies, i.e.,

$$
\frac{d u^{\prime 2}}{d t} \sim-\frac{u^{\prime 3}}{\mathscr{D}_{e}}
$$

(see Ref. 14). Therefore, by substituting (17) and (18) into

$$
\frac{3}{2} u^{\prime 2}=\int_{0}^{\infty} E(k) d k=\frac{3}{2} \int_{0}^{\infty} \phi_{L}(\omega) d \omega,
$$

where the equalities are obtained by definition, one finds $u^{\prime} \mathscr{T}_{L} \sim \mathscr{L}_{e}$, provided $k_{\eta} \gg k_{o}$ and $\omega_{\eta} \gg \omega_{o}$. Although the above spectra are proper only for very large Reynolds number, their result, however, encourages the assumption that $u^{\prime} \mathscr{F}_{L} \rightarrow \mathscr{E}_{e}$.

Combined with $\mathscr{F}_{s} \sim \mathscr{T}_{L}$, we thus propose that

$$
\mathscr{F}_{s} \sim \mathscr{F}_{L} \sim \mathscr{E}_{e} / u^{\prime} \sim \lambda R e_{\lambda} / u^{\prime} \sim\left(t+t_{o}\right),
$$

giving the result of the previous investigators ${ }^{18,27}$ but on a much firmer ground. Note since $\mathscr{T}_{s}=t+t_{o}$, we have $s=\log \left(1+t / t_{o}\right) \quad$ and $\mathscr{T}_{s}\left(t^{\prime}\right)=t_{o} e^{s^{\prime}}=t_{o} e^{s} e^{s^{\prime \prime}}=\mathscr{T}_{s}(t) e^{s^{\prime \prime}}$. Equation (16) thus becomes

$$
\mathscr{F}_{L}(t)=\mathscr{Z}_{s}(t) \int_{0}^{\infty} e^{s^{\prime \prime}} \tilde{f}_{L}\left(s^{\prime \prime}\right) d s^{\prime \prime},
$$

consistent with the assumption $\mathscr{F}_{S} \sim \mathscr{F}_{L}$ and implying a linear growth of $\mathscr{T}_{L}$ in decaying turbulence provided $\tilde{f}_{L}\left(s^{\prime \prime}\right)$ decays faster than $e^{-s^{\prime \prime}}$.

In Figs. 6 and 7, we show $f_{L}$ versus $\left(t^{\prime}-t\right) / \mathscr{T}_{O}$ and $\tilde{f}_{L}$ versus $s^{\prime \prime}=\log \left\{\left(t^{\prime}+t_{o}\right) /\left(t+t_{o}\right)\right\}$, where $\mathscr{F}_{o}$ is the eddy turnover time at $t=0$. Self-prescrvation is observed. Moreover the coefficient becomes exponential at large time separation $s^{\prime \prime}$, i.e. $\tilde{f}_{L} \sim \exp \left(-s^{\prime \prime} / \mathscr{T}^{\prime \prime}\right)$, where $\mathscr{F}^{\prime \prime}$ is a constant, implying an algebraic decay of $f_{L}\left(t, t^{\prime}\right)$ at large time lags 

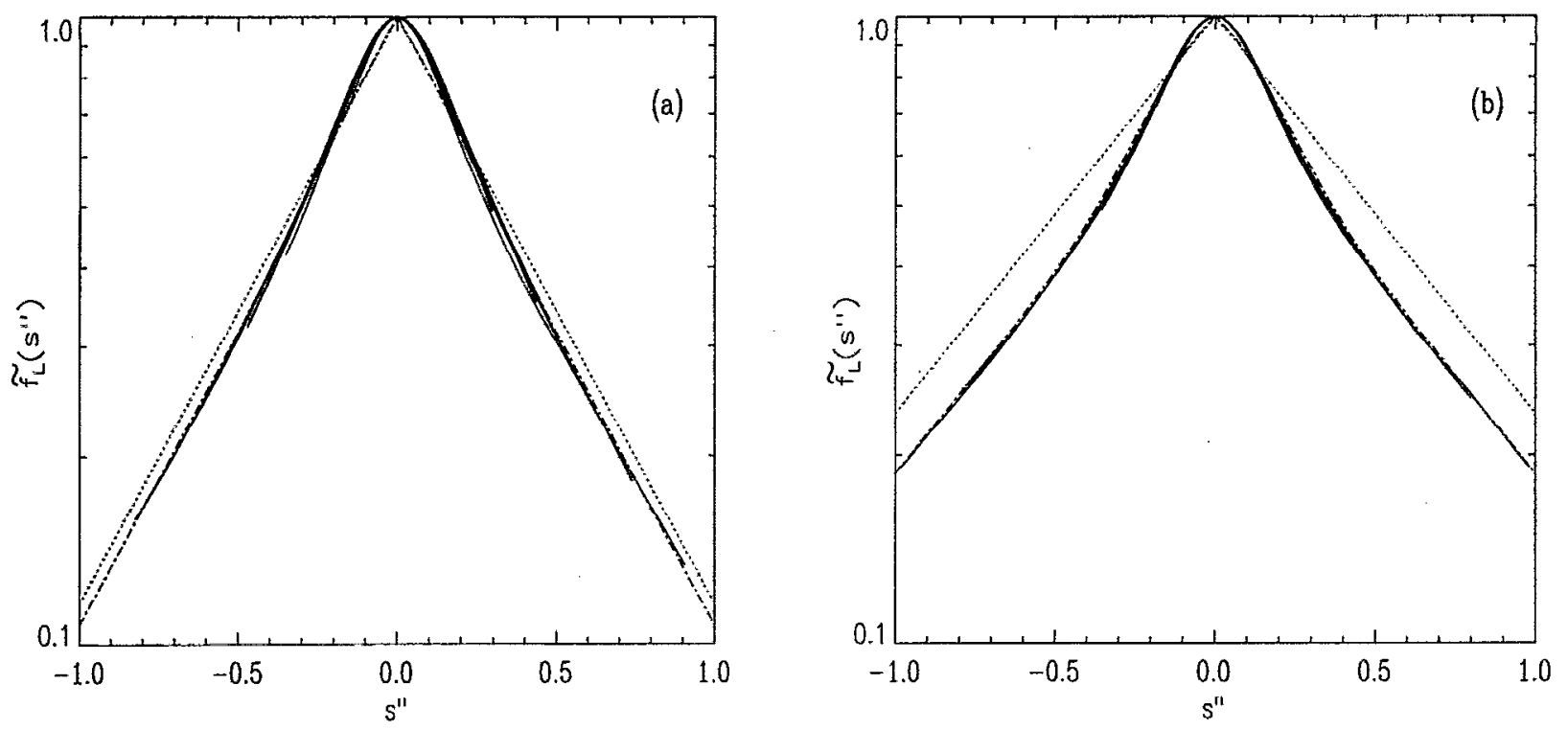

FIG. 7. The self-preserving Lagrangian velocity autocorrelation coefficients $\tilde{f}_{L}$ vs $s^{\prime \prime}=s^{\prime}-s$ for (a) run 1 , (b) run 2 . The dotted lines are the approximating function (26) and the dash-dotted lines are the function (27).

$\left(t^{\prime}-t\right)$ in agreement with the prediction of Saffman. ${ }^{17}$ Also note $\mathscr{T}^{\prime \prime}<1$ (see Table III), implying that $\mathscr{F}_{L}$ exists. Using this exponential tail and equation (21), we can then calculate $\mathscr{F}_{L}$. Defining $\mathscr{E}_{e} \equiv u^{\prime 3} / \epsilon$, we show the calculated result of the ratio of $\mathscr{L}_{e} / u^{\prime} \mathscr{F}_{L}$ in Fig. 8. Note the ratios in the two simulations are different, implying an initial-condition dependence of this ratio.

Finally with the measured $\mathscr{F}_{L}$, we plot $f_{L}$ vs $\left(t^{\prime}-t\right) / \mathscr{F}_{L}(t)$ (Fig. 9) and superpose experimental data of Sato and Yamamoto. ${ }^{30}$ The agreement is not particularly good. The reason may be mainly due to the errors in estimating $\mathscr{T}_{L}$. Because of the difficulty in simulating or measuring the tails of $f_{L}$, the integral timescale $\widetilde{\mathscr{F}_{L}}$ is estimated using a dataset with limited time lags (including those of Sato and Yamamoto). Alternate ways of determining $\mathscr{F}_{L}$, were developed by Squires and Eaton ${ }^{13}$ and by Shlien and Corrsin. ${ }^{2}$ In the present study, however, we take advantage of the selfpreservation of $f_{L}$ and are capable of extending and integrating $f_{L}$ to infinite time lags. $\mathscr{T}_{L}$ determined in such a way thus a tendency to be greater than they would be otherwise. The associated curves in Fig. 9 thus decay more rapidly.

\section{Particle dispersion}

An important application of the self-preserving property of $\tilde{f}_{L}$ discussed in the previous section is its use to predict particle dispersion in a decaying turbulent flow (see Ref. 27).

TABLE III. Turbulence characteristic timescales.

\begin{tabular}{lcccr}
\hline & $n$ & $t_{0}$ & $\Im^{\prime \prime}$ & $\mathrm{b}$ \\
\hline Run 1 & 1.48 & 9.6 & 0.47 & 12 \\
Run 2 & 1.27 & 13.5 & 0.69 & 4 \\
\hline
\end{tabular}

As first derived by Taylor, ${ }^{1}$ the 1-D mean-square displacement of fluid particles, $\left\langle X^{2}(t)\right\rangle$, satisfies the evolution equation,

$$
\frac{d}{d t}\left\langle X^{2}(t)\right\rangle=2 \int_{0}^{t} u^{\prime}(t) u^{\prime}\left(t^{\prime}\right) f_{L}\left(t, t^{\prime}\right) d t^{\prime}
$$

in an isotropic turbulent flow. Applying the self-preserving hypothesis to (22), we obtain

$$
\frac{d}{d t}\left\langle X^{2}(t)\right\rangle=2 t_{o} u_{o}^{\prime 2} e^{(1-n) s} \int_{0}^{s} \exp \left(-(1-n / 2) s^{\prime \prime}\right) \tilde{f}_{L}\left(s^{\prime \prime}\right) d s^{\prime \prime},
$$

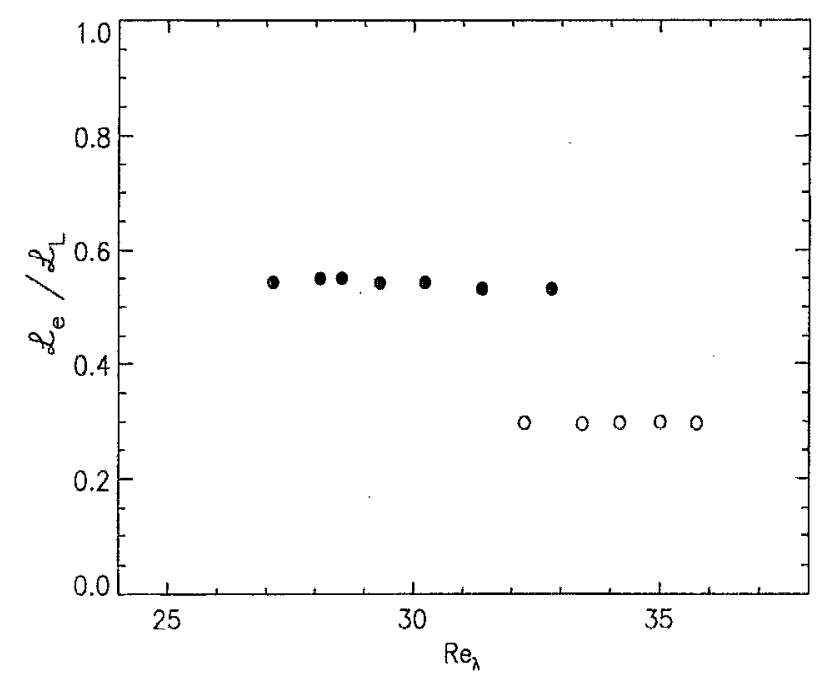

FIG. 8. The ratio of integral lengthscale $\mathscr{L}_{e}$ to $\mathscr{E}_{L}=u^{\prime} \mathscr{P}_{L} \cdot \bullet$, run $\mathrm{I}, \mathrm{O}$, run 2 . 


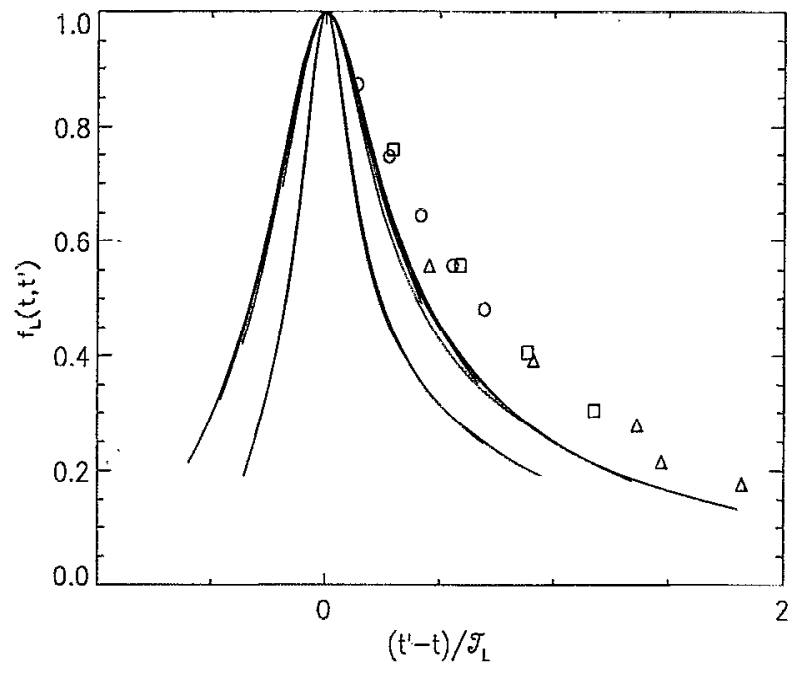

FIG. 9. Here $f_{L}$ vs $\left(t^{\prime}-t\right) / \mathscr{F}_{L}$. The group of curves that decay faster are for run 2. Symbols are for the experimental data of Sato and Yamamoto: $\Delta, R e_{\lambda}=66 ; \square R e_{\lambda}=46 ; O, R e_{\lambda}=25$.

where $u^{\prime 2}(t)=u^{\prime}{ }_{0} 2\left(1+t / t_{o}\right)^{-n}$ is assumed. To check the behavior at small time lags, $s^{\prime \prime} \& 1$, we note that

$$
\int_{0}^{s} \exp \left(-(1-n / 2) s^{\prime \prime}\right) \tilde{f}_{L}\left(s^{\prime \prime}\right) d s^{\prime \prime} \approx s+\emptyset\left(s^{2}\right) ;
$$

and therefore it follows from (23) that

$$
\left\langle X^{2}\right\rangle(t)=u^{\prime 2} t^{2}+\vartheta\left(t^{3}\right) .
$$

When the time lag is large $\left(s^{\prime \prime}\right.$ or $\left.t^{\prime}-t \rightarrow \infty\right)$ and provided the integral in (23) converges, it is easily seen that

$$
\left\langle X^{2}\right\rangle(t) \approx C(n) t_{o}^{2} u_{o}^{\prime 2}\left(1+t / t_{o}\right)^{(2-n)},
$$

where

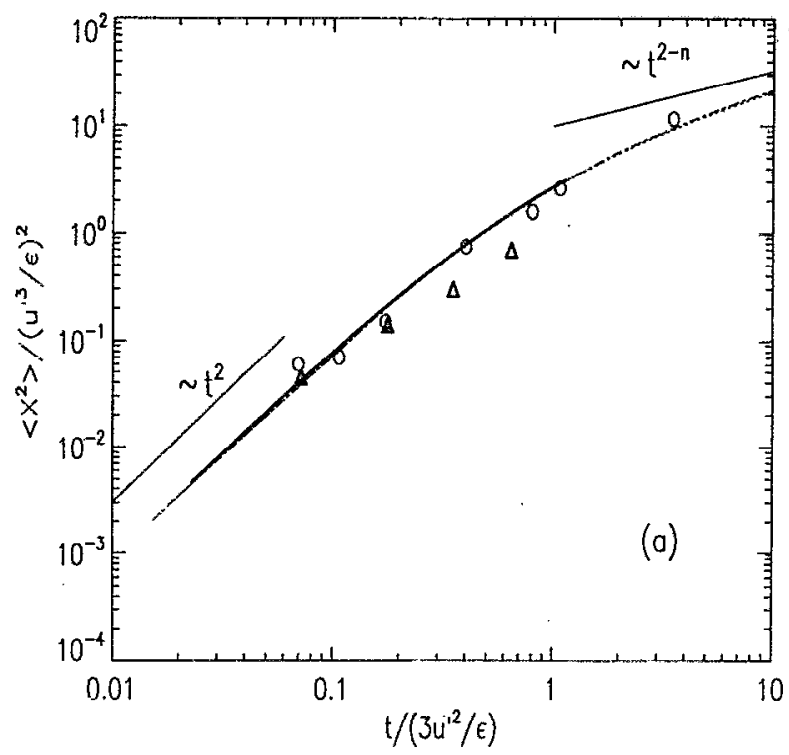

$$
C(n)=\frac{2}{(2-n)} \int_{0}^{\infty} \exp \left(-(1-n / 2) s^{\prime \prime}\right) \tilde{f}_{L}\left(s^{\prime \prime}\right) d s^{\prime \prime}
$$

Note the long-time growth is the same as that of stationary turbulence if $n=1$. Note also that the above anaylsis cannot be applied to stationary turbulence by simply assigning $n=0$, because, in this case, $\mathscr{T}_{s} \sim$ const. $\left(s \sim t / t_{o}\right)$ rather than $\mathscr{T}_{s} \sim t+t_{o}\left(s \sim \log \left(1+t / t_{o}\right)\right)$.

Numerical results for particle dispersion are shown in Fig. 10 with the variables nondimensionalized by flow quantities at $t=0$. Results of the present simulations are presented by solid lines; while the dotted lines are the result of approximating $\tilde{f}_{L}$ in (23) by

$$
\tilde{f}_{L}\left(s^{\prime \prime}\right)=\exp \left(-\frac{s^{\prime \prime}}{\mathscr{T}^{\prime \prime}}\right)
$$

using the measured $\mathscr{P}^{\prime \prime}$ in Table III. This approximation actually overestimates the exponential tails of $\tilde{f}_{L}$ as seen in Fig. 7. The use of

$$
\tilde{f}_{L}\left(s^{\prime \prime}\right)=\frac{1}{1+b}\left(\exp \left(-s^{\prime \prime 2} / 2 s_{o}^{2}\right)+b \exp \left(-s^{\prime \prime} / \mathscr{T}^{\prime \prime}\right)\right)
$$

to correct the asymptote by adjusting the value of $b$ (see Table III) is represented by the dash dotted line in both Fig. 7 and 10 . Both theoretical curves are found in very good agreement with the numerical results. Superposed are experimental data measured by Warhaft ${ }^{7}$ and by Stapountzis et al. ${ }^{6}$ from the interference of passive thermal fields produced by line sources in decaying grid turbulence, which we extract from Fig. 3(b) of Squires and Eaton. ${ }^{13}$ The experimental data was shown to be in good agreement with Anand and Pope's modelling ${ }^{28}$ of a thermal wake (see Fig. 10 of Warhaft ${ }^{7}$ ).

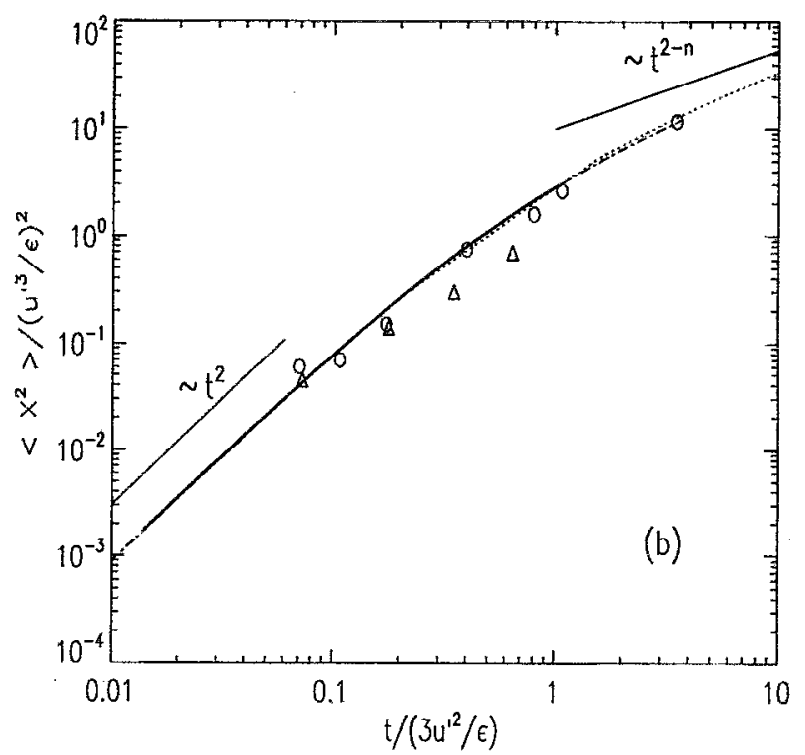

FIG. 10. Particle mean-square displacement for (a) run 1, (b) run 2; $\mathrm{O}$, Warhaft; $\Delta$, Stapountzis, et al. 
Our results, like those of Squires and Eaton, are in good agreement with Warhaft's measurements but slightly above those of Stapountzis et al.

The asymptotic behavior of $\left\langle X^{2}\right\rangle(t) \sim t^{2}$ at small time lags is observed. However, neither Warhaft's data nor ours reaches the long-time asymptotic behavior, i.e. $\left\langle X^{2}\right\rangle(t) \sim\left(1+t / t_{o}\right)^{2-n}$ as $t \gg 1$, as this requires $s=\log \left(1+t / t_{o}\right) \gg 1$ or $t /\left(3 u^{\prime 2} / \epsilon\right) \sim Q(10)$ as suggested by Fig. 10. Because the last few points of the Warhaft data suggest a $\sim t$ behavior, Squires and Eaton' ${ }^{13}$ believe that the asymptotic behavior has been reached with $\sim t$ behavior rather than $\sim t^{2-n}$ behavior because, they conjecture, all length scales are increasing with $t^{1 / 2}$. On the other hand, we find self-preservation of $\tilde{f}_{L}$ in $s^{\prime \prime}$ and, hence, the asymptotic results shown in Fig. 10 (dotted as well as dash dotted curves) even though the various turbulence lengthscales have different time behaviors, i.e.,

$$
\begin{aligned}
& \mathscr{P}_{e} \equiv u^{\prime 3} / \epsilon \sim \lambda R e_{\lambda} \sim t^{1-n / 2}, \\
& \lambda \equiv 15 \nu u^{\prime 2} / \epsilon \sim t^{1 / 2}, \\
& \eta \equiv\left(\nu^{3} / \epsilon\right)^{1 / 4} \sim \lambda \operatorname{Re}_{\lambda}^{-1 / 2} \sim t^{(1+n) / 4} .
\end{aligned}
$$

Thus, we are in agreement with Warhaft's assessment ${ }^{7}$ that the long-time limiting behavior is simply not yet reached but is expected if the experiments or simulations could be extended to even larger times (i.e. as indicated by the theoretical curves in Fig. 10). Note that we are able to obtain asymptotic behavior that is linear in the new, nondimensional time variable but only if we nondimensionalize $\left\langle X^{2}\right\rangle(t)$ appropriately. That is, using (24) and (28), we find that

$$
\frac{d<X^{2}>(t)}{\mathscr{Z}_{e}^{2}} \sim d s
$$

for large time lags.

\section{CONCLUSIONS}

Numerical simulations of decaying, approximately isotropic, homogeneous turbulence have been performed. Lagrangian statistics are obtained by tracking 16,384 or 30,000 fluid particles, using a third-order Taylor series interpolation scheme to estimate the particle velocities. We compute Lagrangian and Eulerian (two-time-one-point and one-timetwo-point) velocity autocorrelation coefficients. The results show that the two-time-one-point Eulerian coefficient is smaller than the Lagrangian coefficient at small time lags, suggesting that the Lagrangian coefficient is more persistent, in agreement with the advection hypothesis proposed by Tennekes in 1975. The two-time-one-point Eulerian coefficient, however, has a longer tail than the Lagrangian coefficient at large time lags. This implies that there is more energy contained within eddies of small frequencies for an Eulerian observer than for a Lagrangian observer. With the observation of a power-law energy decay, we reconsider the classic hypothesis of Batchelor and Townsend including the selfpreservation of Lagrangian velocity autocorrelation. Making the assumption that the turbulent characteristic timescale appearing in the hypothesis $\left(\mathscr{F}_{s}\right)$ is proportional to Lagrangian

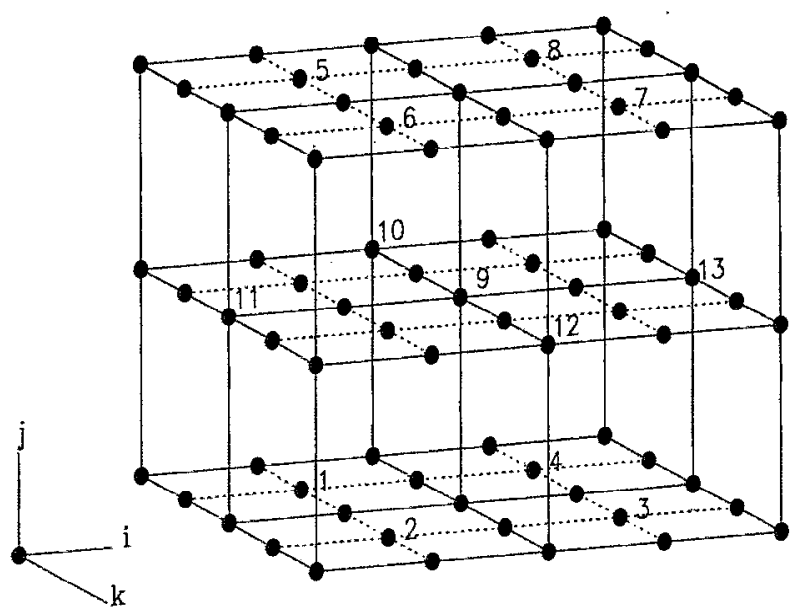

FIG. 11. Grid points used in the modified T13 scheme. Solid lines are grid lines. Dashed lines are also grid lines but are half-integer grid lines in the original T13 scheme of Yeung and Pope.

integral timescale $\left(\mathscr{T}_{L}\right)$ and $u^{\prime} \mathscr{F}_{L} \sim \mathscr{B}_{e} \equiv u^{\prime 3} / \epsilon$, we obtain $\mathscr{T}_{s}=t+t_{o}$. Our numerical results then do indeed show that $f_{L}$ is self-preserving in the new time variable $s=\log \left(1+t / t_{o}\right)$. This self-preservation enables us to predict particle dispersion in decaying turbulence with a power-law energy decay by evoking Taylor's classical work of 1921 . The results of the prediction agree well with the present numerical results and reasonably well with available experimental data.

\section{ACKNOWLEDGMENTS}

We thank Dr. Robert Rogallo for his generosity for providing the basic program for the simulations. This research was performed in part using the Intel Touchstone Delta System operated by Caltech on behalf of the Concurrent Supercomputing Consortium, and supported by the U.S. Air Force Office of Scientific Research under Grant No. AFOSR-910241.

\section{APPENDIX: MODIFIED T13 SCHEME}

The modified T13 method is described below. Suppose a particle at $\mathbf{x}_{p}$ at time $t$ locates within a cube centered at the grid point $x_{9}$ as shown in Fig. 11, the modified T13 algorithm esitmates the particle's velocity as follows

$$
\mathbf{u}\left(\mathbf{x}_{p}(t), t\right) \approx \sum_{i=1}^{13} c_{i} \mathbf{u}\left(\mathbf{x}_{i}, t\right)=\mathbf{u}\left(\mathbf{x}_{p}(t), t\right)+\Theta\left(\Delta \mathbf{x}^{3}\right),
$$

where 


$$
\begin{aligned}
& c_{1}=\left(-x-y-z+y^{2}+x y+x z+y z-x y z\right) / 8, \\
& c_{2}=\left(-x-y-z+y^{2}+x y-x z-y z+x y z\right) / 8, \\
& c_{3}=\left(x-y-z+y^{2}-x y+x z-y z-x y z\right) / 8, \\
& c_{4}=\left(x-y-z+y^{2}-x y-x z+y z+x y z\right) / 8, \\
& c_{5}=\left(-x+y-z+y^{2}-x y+x z-y z+x y z\right) / 8, \\
& c_{6}=\left(-x+y-z+y^{2}-x y-x z+y z-x y z\right) / 8, \\
& c_{7}=\left(x+y-z+y^{2}+x y+x z+y z+x y z\right) / 8, \\
& c_{8}=\left(x+y-z+y^{2}+x y-x z-y z-x y z\right) / 8, \\
& c_{9}=1-\frac{1}{4} x^{2}-\frac{1}{2} y^{2}-\frac{1}{4} z^{2}, \\
& c_{10}=\left(z^{2}-y^{2}\right) / 8, \\
& c_{11}=\left(x^{2}-y^{2}\right) / 8, \\
& c_{12}=c_{10}, \\
& c_{13}=c_{11}, \\
&
\end{aligned}
$$

and

$$
\begin{aligned}
& x \equiv\left(x_{p}-x_{9}\right) / \Delta x, \\
& y \equiv\left(y_{p}-y_{9}\right) / \Delta y, \\
& z \equiv\left(z_{p}-z_{9}\right) / \Delta z .
\end{aligned}
$$

${ }^{1}$ G.I. Taylor, "Diffusion by continuous movements," Proc. London Math. Soc. Ser. 2 20, 196 (1921).

${ }^{2}$ D.J. Shlien and S. Corrsin, "A measurement of Lagrangian velocity autocorrelation in approximately isotropic turbulence," J. Fluid Mech. 62, 255 (1974).

${ }^{3}$ Y. Sato and K. Yamamoto, "Lagrangian measurement of fluid-particle motion in an isotropic turbulent field," J. Fluid Mech. 175, 183 (1987).

${ }^{4}$ M.S. Uberoi and S. Corrsin, "Diffusion of heat from a line source in isotropic turbulence," National Advisory Committee for Aeronautics Reporl No. 1142, 1953.

${ }^{5}$ A.A. Townsend, "The diffusion behind a line source in homogeneous turbulence," Proc. R. Soc. London Ser. A 224, 487 (1954).

${ }^{6}$ H. Stapountzis, B.L. Sawford, J.C.R. Hunt, and R.E. Britter, "Structure of the temperature field downwind of a line source in grid turbulence," J. Fluid Mech. 165, 401 (1986).

${ }^{7} \mathrm{Z}$. Warhaft, "The interference of thermal fields from line sources in grid turbulence" J. Fluid Mech. 144, 363 (1984).
${ }^{8}$ J.J. Riley and G.S. Patterson, "Diffusion experiments with numerical integrated isotropic turbulence," Phys. Fluids 17, 292 (1974).

${ }^{9}$ P.K. Yeung and S.B. Pope, "An algorithm for tracking fluid particles in numerical simulations of homogeneous turbulence," J. Comput. Phys. 79, 373 (1988).

${ }^{10}$ P.K. Yeung and S.B. Pope, "Lagrangian statistics from direct numerical simulations of isotropic turbulence," J. Fluid Mech. 207, 531 (1989).

${ }^{11}$ T. Gotoh, R.S. Rogallo, J.R. Herring, and R.H. Kraichnan, "Lagrangian velocity correlations in homogeneous isotropic turbulence," Phys. Fluids A 5, 2846 (1993).

${ }^{12}$ C.L. Lee, K.D. Squires, J.P. Bertoglio, and J.H. Ferziger, "Study of Lagrangian characteristic times using direct numerical simulation of turbulence," Turb. Shear Flows 6, 58 (1989).

${ }^{13}$ K.D. Squires and J.K. Eaton, "Lagrangian and Eulerian statistics obtained from direct numerical simulations of homogeneous turbulence," Phys. Fluids A 3, 130 (1991).

${ }^{14} \mathrm{~S}$. Corrsin, "Estimates of the relations between Eulerian and Lagrangian scales in large reynolds number turbulence," J. Atmos. Sci. 20, 115 (1963).

${ }^{15} \mathrm{H}$. Tennekes, "Eulerian and Lagrangian time microscales in isotropic turbulence," J. Fluid Mech. 67, 561 (1975).

${ }^{16} \mathrm{~S}$. Corrsin, Proceedings of the Oxford Symposium on Atmospheric Diffusion and Air Pollution (Academic, New York, 1960), p. 162.

${ }^{17}$ P.G. Saffman, "An approximate calculation of the Lagrangian autocorrelation coefficient for stationary homogeneous turbulence," Appl. Sci. Res. A 11, 245 (1962).

${ }^{18}$ G.K. Batchelor and A.A. Townsend, "Turbulent diffusion," in Surveys in Mechanics, edited by G.K. Batchelor and H. Bondi (Cambridge University Press, Cambridge, 1956), p. 352.

${ }^{19}$ R.S. Rogallo, "Numerical experiments of homogeneous turbulence," NASA Technical Memorandum, 81315, 1981.

${ }^{20}$ S.A. Orszag, "Numerical simulation of incompressible flows within simple boundaries. L. Galerkin (spectral) respresentations," Stud. Appl. Math. L, 293 (1971).

${ }^{21}$ M.J. Huang and A. Leonard, "Power-law decay of homogeneous turbulence at low Reynolds numbers," Phys. Fluids 6, 3765 (1994).

${ }^{22} \mathrm{~V}$. Eswaran and S.B. Pope, "An examination of forcing in direct numerical simulations of turbulence," Comput. Fluids 16, 257 (1988).

${ }^{23}$ S.S. Girimaji and S.B. Pope, "Material-element deformation in isotropic turbulence," J. Fluid Mech. 220, 427 (1990).

${ }^{24}$ W.K. George, "The decay of homogeneous isotropic turbulence," Phys. Fluids A 4, 1492 (1992).

${ }^{25}$ J.O. Hinze, Turbulence (McGraw-Hill, New York, 1975).

${ }^{26} \mathrm{Th}$. von Karman and L. Howarth, "On the statistical theory of isotropic turbulence," Proc. R. Soc. London Ser. A 164, 192 (1938).

${ }^{27} \mathrm{Y}$. Sato and $\mathrm{K}$. Yamamoto, "Empirical equations for the structure of isotropic turbulence," J. Chem. Eng. Jpn. 16, 273 (1983).

${ }^{28} \mathrm{M}$.S. Anand and S.B. Pope, "Diffusion behind a line source in grid turbuIence," Turb. Shear Flows 4, 41 (1985). 